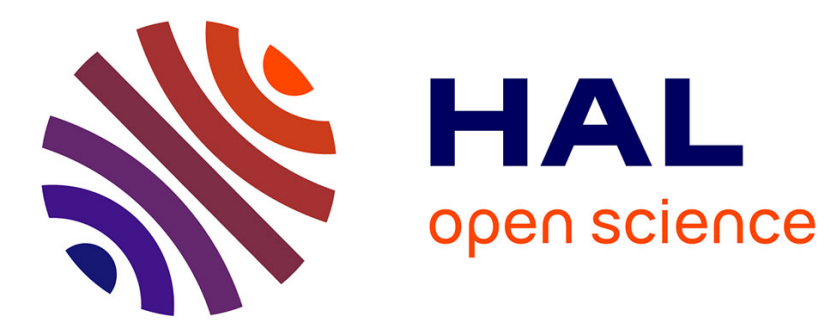

\title{
Nonlinear spectral shaping and optical rogue events in fiber-based systems
}

\author{
Kamal Hammani, Bertrand Kibler, Julien Fatome, Sonia Boscolo, Goëry \\ Genty, John M. Dudley, Guy Millot, Christophe Finot
}

\section{- To cite this version:}

Kamal Hammani, Bertrand Kibler, Julien Fatome, Sonia Boscolo, Goëry Genty, et al.. Nonlinear spectral shaping and optical rogue events in fiber-based systems. Optical Fiber Technology, 2012, 18, pp.248-256. 10.1016/j.yofte.2012.06.009 . hal-00699104

\section{HAL Id: hal-00699104 https://hal.science/hal-00699104}

Submitted on 19 May 2012

HAL is a multi-disciplinary open access archive for the deposit and dissemination of scientific research documents, whether they are published or not. The documents may come from teaching and research institutions in France or abroad, or from public or private research centers.
L'archive ouverte pluridisciplinaire HAL, est destinée au dépôt et à la diffusion de documents scientifiques de niveau recherche, publiés ou non, émanant des établissements d'enseignement et de recherche français ou étrangers, des laboratoires publics ou privés. 


\title{
Nonlinear spectral shaping
}

\section{and optical rogue events in fiber-based systems}

\author{
Kamal Hammani $^{1, !}$, Bertrand Kibler ${ }^{1}$, Julien Fatome ${ }^{1}$, Sonia Boscolo ${ }^{2}$, \\ Goery Genty $^{3}$, John M. Dudley ${ }^{4}$, Guy Millot ${ }^{1}$ and Christophe Finot ${ }^{1, *}$ \\ ${ }^{1}$ Laboratoire Interdisciplinaire Carnot de Bourgogne, UMR 6303 CNRS-Université de \\ Bourgogne, 9 avenue Alain Savary, BP 47870, 21078 Dijon Cedex, France \\ ${ }^{2}$ Photonics Research Group, Aston University, Birmingham, B47ET, United Kingdom \\ ${ }^{3}$ Tampere University of Technology, Optics Laboratory, FI-33101 Tampere, Finland \\ ${ }^{4}$ Institut FEMTO-ST, UMR 6174 CNRS-Université de Franche-Comté, 25030 Besançon, \\ France \\ 'now at the Optoelectronics Research Center, University of Southampton, United Kingdom \\ *Corresponding author: \\ E-mail address: christophe.finot@u-bourgogne.fr \\ Tel.: +33380395926
}




\begin{abstract}
:
We provide an overview of our recent work on the shaping and stability of optical continua in the long pulse regime. Fibers with normal group-velocity dispersion at all-wavelengths are shown to allow for highly coherent continua that can be nonlinearly shaped using appropriate initial conditions. In contrast, supercontinua generated in the anomalous dispersion regime are shown to exhibit large fluctuations in the temporal and spectral domains that can be controlled using a carefully chosen seed. A particular example of this is the first experimental observation of the Peregrine soliton which constitutes a prototype of optical rogue-waves.
\end{abstract}

(C) 2012 Elsevier

Keywords: Fiber optics; supercontinuum ; pulse shaping ; extreme statistics ; optical solitons 


\section{Introduction}

Nonlinearity in single-mode optical fibers has often been regarded as a detrimental effect for the transmission of pulsed signals. However, when properly managed, nonlinearity offers great opportunity to reshape the optical spectrum of coherent light and expand its bandwidth to several hundreds of nanometers. For example, in the early 1990's, supercontinuum (SC) generated in optical fibers has found several practical applications in dense wavelength-division multiplexed communications [1]. The advent of photonic crystal fibers (PCF) in the late 90's has considerably widened the field of applications: by means of accurate control of the periodic arrangement of air holes and silica rods, stronger confinement of the optical field can be achieved so that the nonlinear response is enhanced by more than an order of magnitude compared to conventional fibers. PCFs also offer the crucial advantage of enabling precise engineering of the dispersive properties so that a zero dispersion wavelength (ZDW) well below the ZDW of bulk silica $(1.3 \mu \mathrm{m})$ has become technologically feasible (indeed now commonplace). This has paved the way for efficient nonlinear processes at the wavelengths of Ytterbium technologies $(1.06 \mu \mathrm{m})$ and within the tuning range of Ti-Sapphire lasers [2]. With optical spectra routinely spanning an octave, SCs have found many disruptive applications and have stimulated numerous breakthroughs in optical coherence tomography [3], metrology and spectroscopy [4], biophotonics, and other fields of science.

The physics of the continuum generation is generally complex and crucially depends on the dispersion properties at the pump wavelength. However, chromatic dispersion is not the only parameter affecting the output spectrum, the input pulse duration, peak power and pulse shape also strongly influence the output and further increase the richness and diversity of the nonlinear 
propagation dynamics. In this paper, we choose to focus on how these parameters influence the stability characteristics of SC spectra and how they can be controlled in different regimes. In this respect, the performance of normally dispersive fibers is seen as superior to that of anomalousdispersion fibers, where large shot-to-shot fluctuations are seen both in the temporal and spectral domains [5]. In this context, the observation of rare but intense spikes of light [6] in the anomalous-dispersion regime has initiated the field of optical rogue waves and has provided a new perspective for the analysis of SC stability. This, in turn, has highlighted or confirmed the crucial role of nonlinear coherent structures such as fundamental solitons $[7 ; 8]$, breathers $[9 ; 10]$ and rational solitons such as the Peregrine soliton [11].

Several review articles and books are now available to understand the physical mechanisms driving the continuum formation and its modeling using the nonlinear Schrödinger equation (NLSE) in its extended form $[12 ; 13 ; 14 ; 15 ; 16]$. Therefore it is not our intention here to present an additional comprehensive or analytical description of the nonlinear dynamics of pulses propagating in an optical fiber, but rather to provide an overview of our recent numerical and experimental contributions to the field of SC generation in both the normal and anomalous dispersion regimes. To this end, we restrict ourselves to fibers with a constant longitudinal dispersion profile and to the long pulse regime (i.e. picosecond pumping or longer). Significantly, the results presented in this paper are mostly obtained at telecommunication wavelengths and with commercially available components and fibers.

In the first part of this paper, we emphasize how the normal dispersion regime is particularly well-suited for nonlinear pulse shaping, with the output pulse shape being directly controlled by adjustment of the initial amplitude and phase [17]. In the second part, we show that in the 
anomalous dispersion regime, soliton dynamics govern the temporal and spectral features of the generated SC resulting in large shot-to-shot fluctuations and associated with the emergence of extreme events. We then discuss a possible experimental solution to efficiently harness the observed fluctuations. This particular setup further allows us to experimentally generate a specific solution of NLSE which is considered as a prototype of freak events known as the Peregrine soliton.

\section{Highly coherent supercontinuum and nonlinear spectral shaping in normally dispersive fibers}

Due to the Kerr nonlinearity of silica, the propagation of intense ultrashort pulses in optical fibers is strongly affected by self-phase modulation (SPM) [18]. The resulting nonlinear phase directly depends on the temporal gradient of the pulse intensity profile and leads to broadening of the optical spectrum. For an ideal dispersionless fiber, this broadening would linearly increase with the propagation distance and the temporal intensity profile would remain unchanged. However, in a real fiber, the impact of chromatic dispersion cannot be ignored, especially when the spectral bandwidth becomes substantial, and taking dispersion into account leads to radically different nonlinear dynamics depending on the dispersion regime.

In this section we consider propagation in the regime of normal dispersion. In this case, the chirp induced by the dispersion combines with the nonlinear chirp from SPM, in such as way as to enhance the temporal broadening. As a consequence, the pulse peak power progressively drops and the spectral broadening saturates $[19 ; 20]$. During propagation, the temporal intensity profile also experiences strong reshaping as illustrated in Fig. 1(a2) where an initial hyperbolic secant pulse becomes gradually close to a parabolic shape before undergoing wave-breaking phenomenon [21], and subsequently evolving into a trapezoidal-like pulse profile. The stage of 
wave-breaking occurs when the portions of the pulse with higher instantaneous frequencies temporally overlap with pulse portions having much lower instantaneous frequencies $[20 ; 21$; 22]. In the spectral domain, wave-breaking manifests itself as the generation of new frequencies and the appearance of sidelobes in the spectrum as seen both in the numerical simulations of Fig. 1(a1) and experimental results shown in Fig. 2(a).
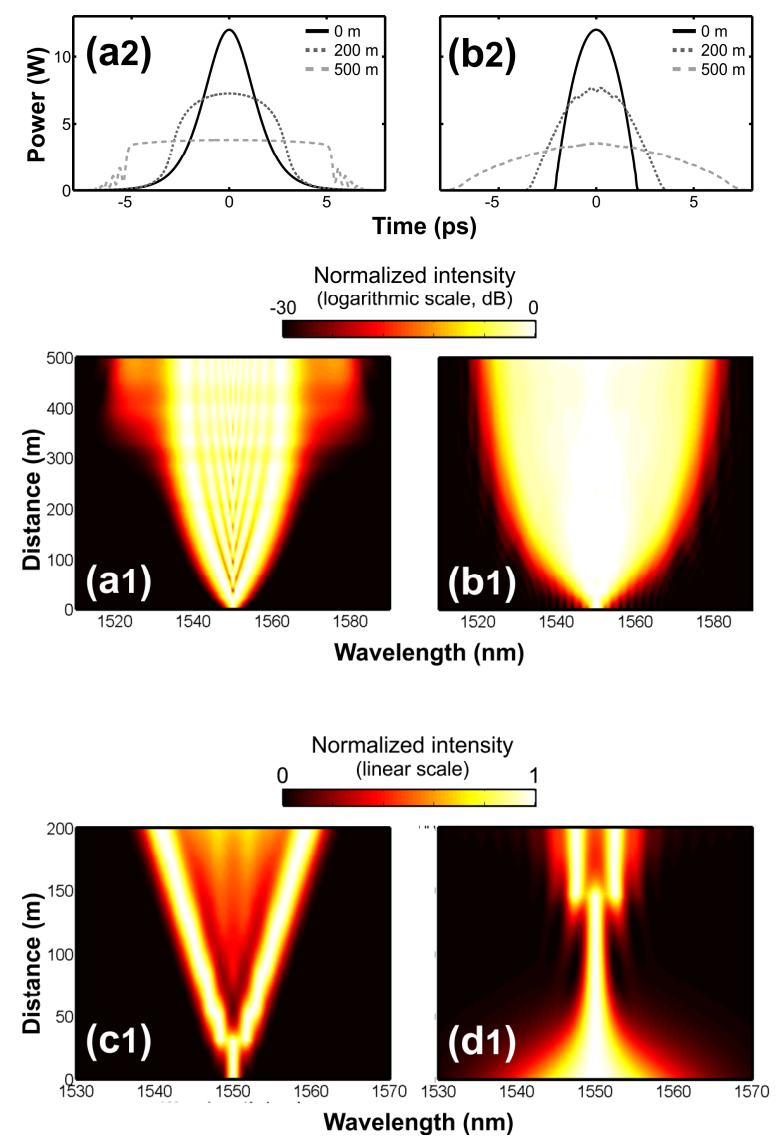

Figure 1: Evolution of different initial pulses in a normally dispersive fiber: transform-limited pulse with hyperbolic secant, parabolic and triangular intensity profiles (subplots a, b, c respectively) and linearly chirped Gaussian pulse (subplot d). Subplots (1) show the longitudinal evolution of the spectral intensity profile, and subplots (2) show the temporal intensity at different stages of propagation. The initial pulses have a full-width at half maximum (FWHM) duration of 3 ps with an input peak power of $12 \mathrm{~W}$ and propagate in a highly non-linear fiber with a non-linear coefficient of $10 / \mathrm{W} / \mathrm{km}$ and a dispersion coefficient of $-0.5 \mathrm{ps} / \mathrm{nm} / \mathrm{km}$. The results are obtained by numerical integration of the standard NLSE. 
As the nonlinear dynamics involved here depends crucially on the input pulse features, it is possible to nonlinearly shape the output spectrum through accurate control of the input pulse temporal intensity or/and phase profiles. It is worth noting that this type of approach can be flexibly applied to generate spectral bandwidth exceeding significantly than that of the input pump pulse. This is obviously not possible with conventional linear shaping techniques where one cannot shift energy outside the initial pulse bandwidth [17]. For example, the use of initial parabolic-shaped pulses allows to prevent optical wave-breaking [23; 24] (see Fig. 1(b) and 2(b) for experimental evidence) as well as to generate a highly flat continuum with enhanced spectral energy density. The use of an initial triangular temporal profile on the other hand leads to the symmetric splitting of the pulse spectrum into two peaks of equal magnitude [25] (Figs. 1(c) and 2(c)), which can be advantageous for the realization of optical functions such as frequency conversion [26] or pulse doubling [27]. The input temporal phase profile also has a strong impact on the pulse evolution in the fiber and, as such, allows the generation of more advanced pulse shapes. For example, an initial Gaussian pulse with a parabolic phase profile (linear chirp) will passively reshape itself into a triangular intensity profile at a given stage of the propagation [25; 28]. Another striking example demonstrating the impact of the input phase profile is the process of spectral recompression where, contrary to what might be expected based on simple considerations, SPM leads to energy concentration at the center of the pulse spectrum $[29 ; 30$; 31; 32] (Figs. 1(d) and 2(d)). 

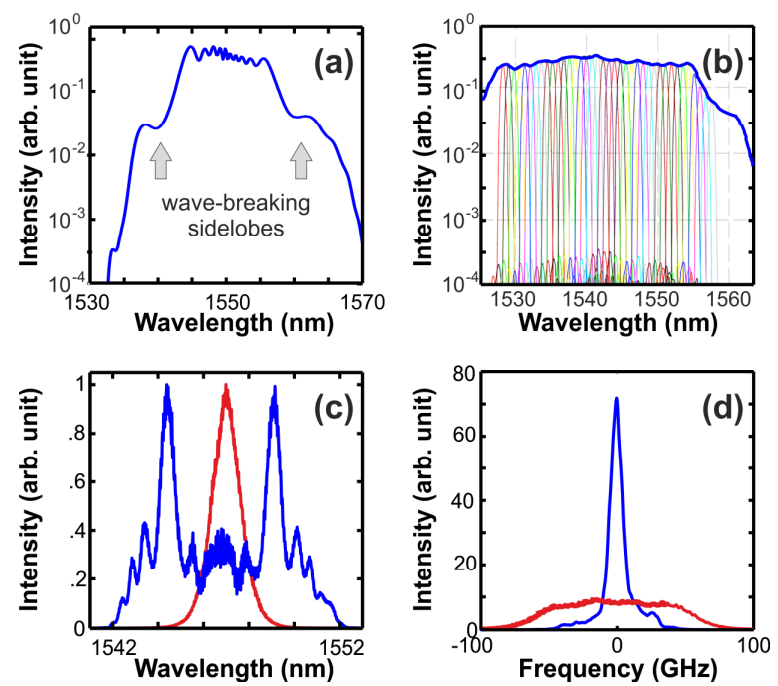

Figure 2: Experimental results of the propagation of a hyperbolic secant pulse in a dispersion-shifted fiber, of a parabolic pulse in a highly nonlinear fiber followed by spectral slicing (the different channels are plotted), of a triangular pulse and of a Gaussian pulse with a parabolic phase (subplots a, b, c and d respectively). Details of the different experimental configurations can be found in [20], [23], [25] and [32] respectively.

Normally dispersive fibers are undoubtedly advantageous compared to anomalous fibers as far as the coherence of the generated continuum is concerned. Specifically, nonlinear propagation in the normal dispersion regime inhibits modulation instability (MI) phenomena to occur, which reduces significantly spontaneous amplification of noise and thereby shot-to-shot fluctuations. Consequently, a high degree of coherence is maintained as illustrated in Fig. 3, where we plot the results of a few hundreds of numerical simulations with distinct input noise seeds. Such a high level of stability is associated with a single temporal coherent structure that is clearly attractive for all-optical pulse processing in highly nonlinear fibers [17] or the generation of SC covering the entire C-band of optical telecommunications. Indeed, several studies have reported that such continua can be sliced into several tens of high-quality high-repetition-rate picosecond channels where isolated spectral lines of the $10-\mathrm{GHz}$ or $40-\mathrm{GHz}$ resulting comb provide several hundreds of continuous waves $[16 ; 23 ; 33]$. The high degree of coherence of the generated spectra further enables efficient pulse compression with very high compression ratio as the nonlinear chirp 
induced by SPM can be compensated to a large extent by an optical fiber providing anomalous dispersion $[26 ; 34 ; 35 ; 36]$ or by a pair of diffraction gratings $[34 ; 37 ; 38]$.
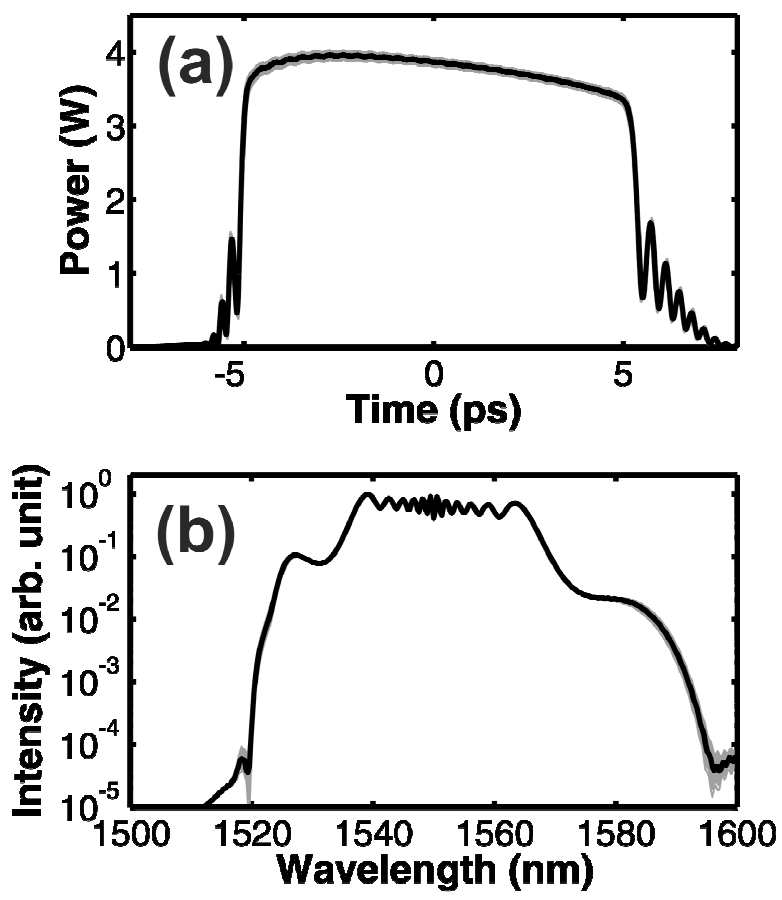

Figure 3: Superposition of 200 simulations of the output temporal (a) and spectral (b) intensity profiles of a hyperbolic secant pulse ( 3 ps FWHM duration, $10 \mathrm{~W}$ peak power) propagating in a 500-m-long highly nonlinear fiber (nonlinear coefficient $10 / \mathrm{W} / \mathrm{km}$, dispersion $-0.5 \mathrm{ps} / \mathrm{nm} / \mathrm{km}$, dispersion slope 0.01 $\mathrm{ps}^{2} / \mathrm{nm} / \mathrm{km}$ at $1550 \mathrm{~nm}$ ). The results are obtained by numerical integration of the NLSE with third-order dispersion, including the quantum noise as well as $1 \%$ fluctuations on the input pump. The different shots are plotted in grey color and the average curve is plotted with a solid black line. Grey and black curves nearly coincide.

This technique has been efficiently exploited in PCFs where dispersion characteristics can be accurately controlled so as to obtain a fiber with normal dispersion at all wavelengths [39] that enables the generation of highly broadband coherent spectra spanning more than several hundreds of nanometers. Such spectra are particularly attractive from the perspective of pulse compression down to duration as short as a few optical cycles after compensation of the nonlinear phase [40; 41]. 
Finally, we would like to point out that the benefits of the regime of normal dispersion in terms of nonlinear pulse shaping can be further enhanced by the additional presence of optical gain. Indeed, regardless of its initial properties, any pulse in this case tends asymptotically towards a parabolic intensity profile both in the temporal and spectral domains, combined with a highly linear chirp. The formed pulse in the amplifier then propagates in a self similar manner, experiencing an exponential increase of its amplitude, duration and spectral bandwidth. Selfsimilar parabolic pulses not only can tolerate strong nonlinearity without wave breaking, but can also be compressed to shorter pulse of very high quality [36; 42]. This makes parabolic pulse amplification an attractive alternative to chirped pulse amplification techniques at moderate energies and is beneficial for generation of high quality continua [43; 44].

\section{Supercontinuum stability in the anomalous dispersion regime and extremes events}

In this section, we review the main properties of SC generated in the anomalous dispersion regime of a nonlinear fiber.

\section{Supercontinuum stability}

The stability of the continuum obtained in the normal regime of dispersion is generally not preserved in the anomalous dispersion region (in fact the stability breaks in the vicinity of the ZDW regardless of the dispersion sign). This is because in the anomalous regime, the SC properties change significantly from shot to shot both in the temporal and spectral domains. The spectral phase fluctuations have been the subject of intense investigations as early as 2000 [45]. 
But it is only at the end of the year 2007 that an article published by Solli et al has reported the experimental observation of significant fluctuations in the temporal domain [6]. By spectrally filtering the most red-shifted part of the SC spectrum generated in a PCF pumped in the regime of anomalous dispersion, Solli et al have analyzed the statistical fluctuation of the corresponding filtered temporal intensity and found that a highly-skewed distribution that strongly differs from the Gaussian shape. By analogy with the typical L-shaped distribution observed for extreme events, the intense and extremely rare outliers of the distribution were named "optical rogue waves". Since then, the subject has become the focus of intense international research in the optical community by the large number of studies published in the last three years. This enthusiasm has indeed largely surpassed the confines of SC generation in PCFs and optical rogue waves have been reported in various nonlinear systems including filamentation [46], optical amplifiers driven by incoherent pumps [47; 48], generation of SC in non-silica materials [49], telecommunications transmission systems [50] or optical feedback systems $[51 ; 52 ; 53 ; 54]$.

An illustration of the temporal and spectral instability of the continuum generated in the anomalous dispersion region is shown in Fig. 4, which shows the results of NLSE numerical simulations similar to those in Fig. 3, but with anomalous dispersion. The nonlinear pulse dynamics is dominated by an initial stage of MI followed by soliton dynamics with splitting of the ultrashort coherent structure due to third-order dispersion. We note that similar splitting would be produced by the intra-pulse Raman effect. Pulse splitting results in the emergence of several ultrashort solitons with significant shot-to-shot amplitude and timing jitters (Fig. 4(a)) which prevents this type of SC to be sliced in multiple channels for WDM applications. The 
spectral intensity profile (Fig. 4(b)) is significantly broader than those typically obtained in fibers with normal dispersion but also suffers from large fluctuations.
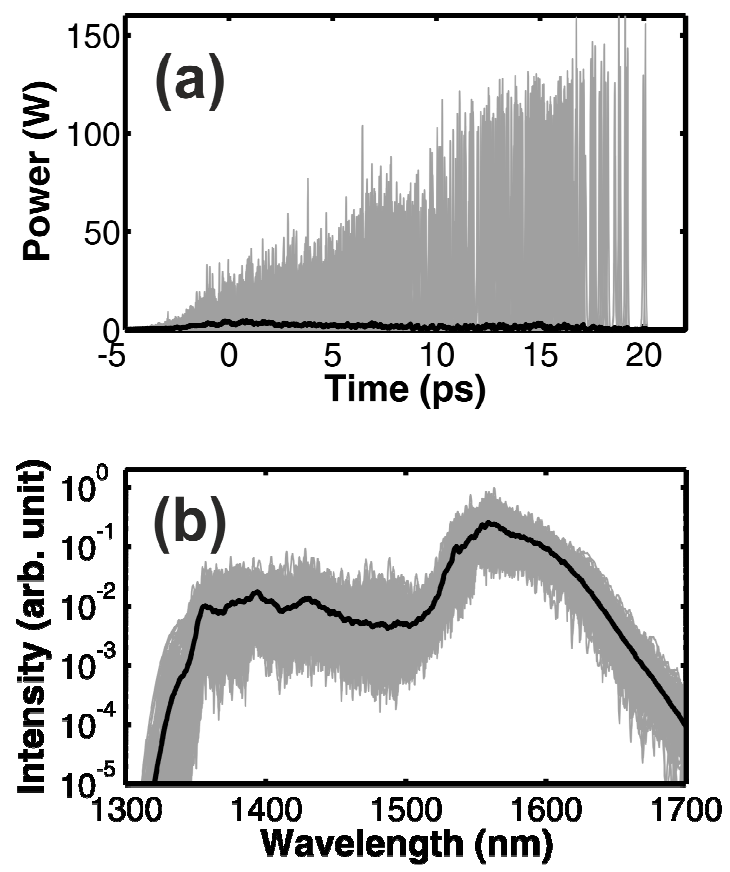

Figure 4: Superposition of 200 simulations of the ouput temporal (a) and spectral (b) intensity profiles of a secant hyperbolic pulse (FWHM duration of $3 \mathrm{ps}$, peak power of $10 \mathrm{~W}$ ) propagating in a 500-m highly nonlinear fiber (nonlinear coefficient $10 / \mathrm{W} / \mathrm{km}$, dispersion $0.5 \mathrm{ps} / \mathrm{nm} / \mathrm{km}$, dispersion slope 0.01 $\mathrm{ps} / \mathrm{nm} / \mathrm{km}$ at $1550 \mathrm{~nm}$ ). Results are obtained by the numerical integration of the standard NLSE with third-order dispersion and include the quantum noise as well as $1 \%$ of the fluctuations on the input pump. The different shots are plotted in grey and the average curve is plotted with a solid black line.

In order to illustrate quantitatively the fluctuations inherent to anomalous dispersion pumping, we present in Fig. 5 results of numerical simulations that take into account the full dispersive and nonlinear responses of the fiber through the integration of the generalized NLSE. Specifically, we show that independently of the fiber characteristics (fiber with one or two ZDWs) $[55 ; 56]$ large fluctuations are present on the red side of the generated SC with associated 
L-shaped histograms (see subplots 2), when a filter is used to isolate those fluctuations. We also stress that significant fluctuations can occur for normal pumping when the initial pulse spectrum lies close to the ZDW (see Fig. 5c) [55; 56].
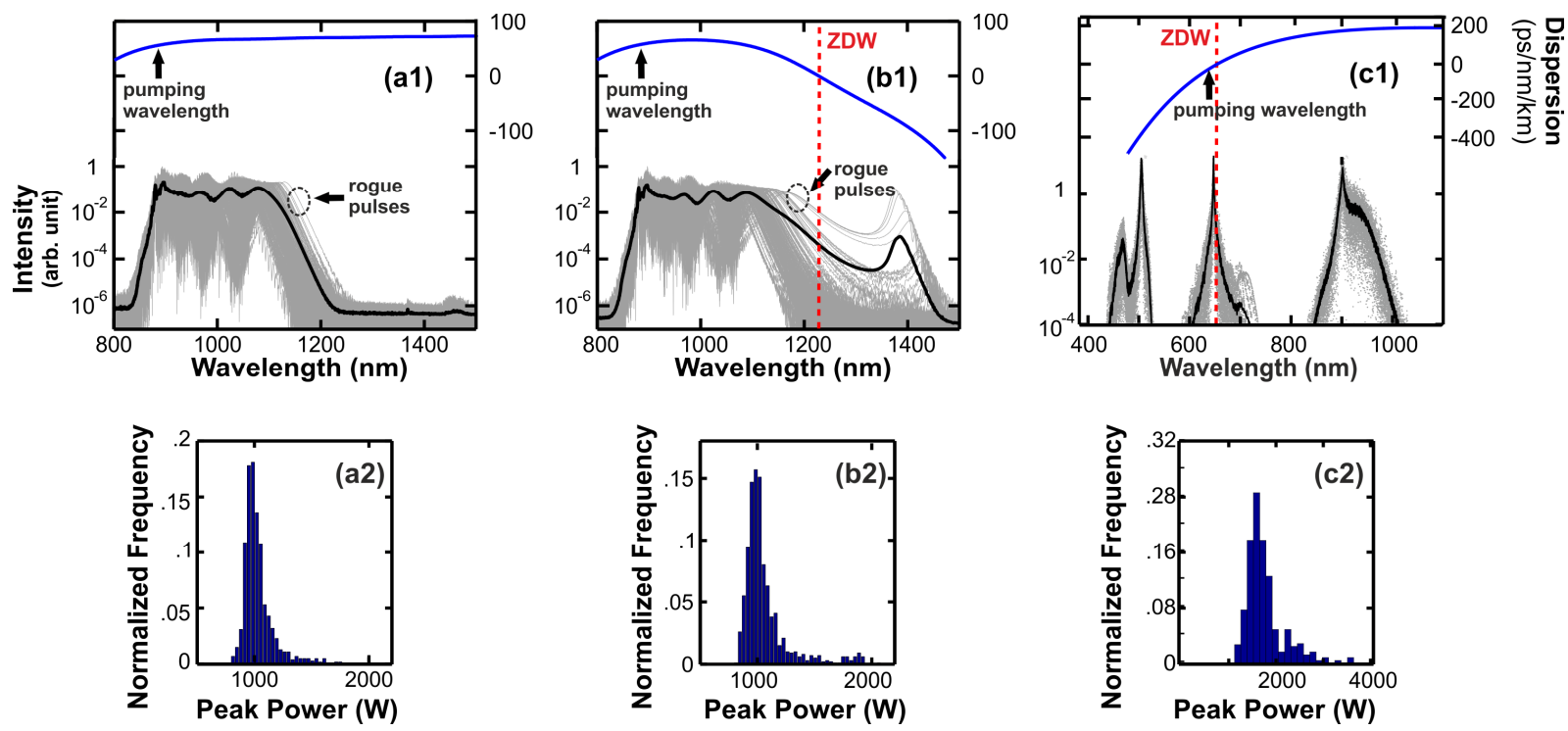

Figure 5: Superposition of 200 simulations of the pulse spectrum after propagation in a fiber with a single ZDW pumped in anomalous dispersion (subplots a), a fiber with two ZDWs and pumped in the anomalous dispersion regime (subplots b), and a fiber pumped in the normal dispersion regime and where the broadening process is governed by fourth-order modulation instability (subplots c). Details of the fiber parameters, input pulses can be found in publications [57] and [58] respectively. The results are obtained by the numerical integration of the generalized NLSE.

\section{Optical rogue solitons}

The main questions arising from the pioneering observations of rogue waves in optical systems are directed towards the physical nature of the extreme events and the mechanisms behind their emergence. A careful analysis of various configurations has now led to the conclusion that the most red-shifted events detected in the original experiments of Solli et al are fundamental 
solitons shifted in frequency by the intra-pulse Raman response of silica [59]. As an example, Fig. 6 shows the spectrogram of an extreme event obtained in a SC driven by fourth-order modulation instability [58]. One can clearly identify the signature of the extreme event in the form of a transform-limited coherent soliton structure that emerges at long wavelengths. Because such giant solitons are fully coherent and persist once they form, they are now generally referred to as "rogue solitons" [60].
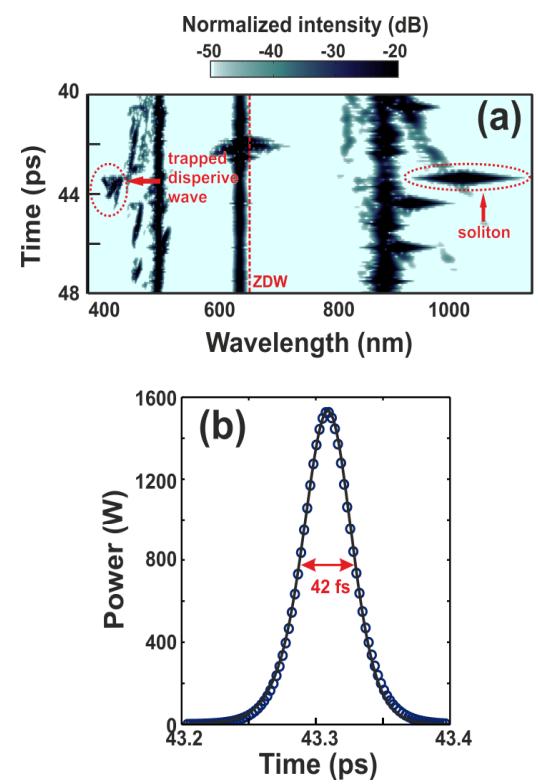

Figure 6: (a) Spectro-temporal representation of a rogue event occurring in a SC driven by fourth-order modulation instability (cf. Fig. 5c). (b) Detail of the temporal structure of the most frequency red-shifted event (solid line) compared with the shape of a fundamental soliton. Details of the numerical simulations can be found in [58].

A physical process involved in the emergence of extreme solitons is the process of temporal collisions between the substructures generated by splitting of an initial pulse. When two ultrashort substructures collide, a temporal peak appears intermittently, thus reproducing one of the peculiar features of the infamous oceanic freak waves [61]: their unexpected appearance and 
decay. Such collisions are favored by convective mechanisms such as the third-order dispersion or the Raman response of silica [62]. A dedicated care should be provided to their detection by a frequency offset filter that can in itself create an artifact [63] and may not see these collision events directly $[64 ; 65]$ as the emerging temporal spike of light does not correspond to the most red-shifted event. In the process of collision, an energy exchange also occurs, leading to the progressive growth of a giant soliton, where most of the energy of the system will eventually concentrate [66; 67]. It is worth noting here that fluctuations do not develop only on the long wavelength side of the SC, but also on the short wavelength edge of the supercontinuum with a statistical distribution that deviates from a Gaussian statistics $[58 ; 68]$ as a result of pulse trapping by the redshifted solitons [69] .

\section{Spontaneous modulation instability and Akhmediev breathers}

Whilst SC dynamics are now well-understood and rogue events can be explained through the prism of optical solitons [8], the temporal analysis of the fluctuations associated with anomalous pumping has led to reconsider the onset of SC generation where higher-order linear and nonlinear effects do not play an extensive role and pulse propagation can be described in the framework of the standard NLSE. In fact, the stage of MI that governs the initial spectral broadening can be described analytically within the framework of the Akhmediev breather formalism. Akhmediev breathers are particular solutions of the NLSE derived by Akhmediev and Korneev in 1986 which describe the single growth-return cycle of an initially weak sinusoidal modulation [9]. Numerical simulations and experiments have indeed confirmed that the properties of SC at the early stages of propagation can be accurately described in terms of multiple breathers corresponding to the excitation of the entire MI gain bandwidth [10]. In 
particular, the characteristics observed at the point of maximum temporal compression can be estimated by considering the properties of the breather corresponding to the frequency of the MI gain peak and whose spectrum exhibits a characteristic triangular shape on a log-log scale [10].
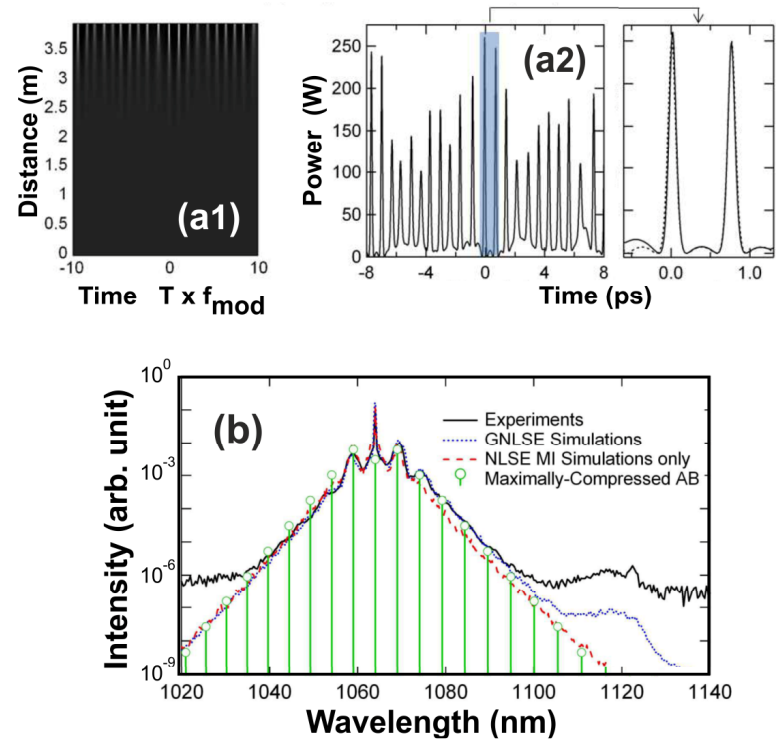

Figure 7: (a) Single shot NLSE simulations of the longitudinal evolution of a CW field undergoing spontaneous MI (subplot a1) and details of the temporal profile at $3.5 \mathrm{~m}$ propagation distance (subplot (a2)). The shaded region in the temporal trace is shown in detail in the rightmost figure comparing simulations (solid line) with the Akhmediev breather solution calculated for a modulation frequency corresponding to peak MI gain (dashed line). (b) Comparison between experiments (solid black line), numerical simulations using the full GNLSE (blue dashed line), numerical simulations using the NLSE only (red dashed line), and the calculated spectrum of the maximally-compressed Akhmediev breather (green open circles connected to the horizontal axis by straight lines from zero). Figures adapted from [10].

\section{Generation of highly coherent supercontinuum in the anomalous dispersion regime and observation of the Peregrine soliton}

\section{Seeded generation}


The various examples of SC generation described in the previous section show that the control of the spectral dynamics, shape and stability and coherence properties is in general a complex issue. In the normal dispersion regime, changing the temporal intensity or phase profile of the input pulse can provide a direct means of tailoring the continuum. On the contrary, in the anomalous dispersion regime, the SC dynamics is governed by solitonic effects that are more difficult to harness.

One way to limit the degradation of the SC coherence is to use very short segments of fiber or tapered fibers $[5 ; 70]$. An alternative solution is to reduce the spontaneous instabilities that may emerge in the first stages of propagation. In this paper, we do not consider the case when the pump itself is only partially coherent, which would require consideration on an incoherent modulational instability [48]. Here the pump is a coherent signal only affected by quantum noise and a small level of input amplitude fluctuations. By imposing an input seed located in the frequency band of the instabilities, one can expect that this deterministic seed will prevail over the spontaneous effects so that enhanced stability and coherence can be ensured. Such an approach has been used in various configurations, in both numerical [71; 72] (Fig. 8(a)) and experimental $[73 ; 74 ; 75]$ works in order to control the stability and spectral extent of the generated continuum (Fig. 8(b)). Other straightforward applications to optical parametric amplification and frequency conversion [76] as well as the all-optical generation of stable pulse trains with very high repetition rates $[34 ; 77]$ have also benefited from the enhanced stability resulting from seeded parametric processes. An accurate spectral study has confirmed that the seeded generation can be well described by the mathematical formalism introduced by Akhmediev [78]. 

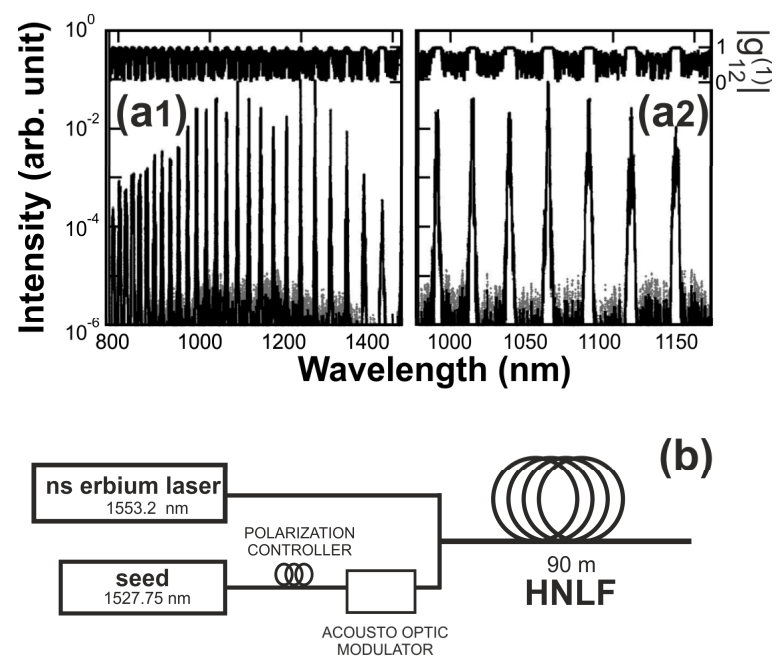

(b)

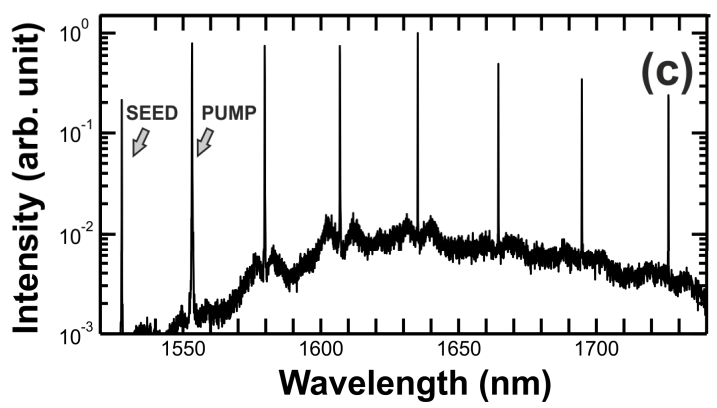

Figure 8: (a1) Simulations results of SC generated by 20 ps pulses with modulation at $12.5 \mathrm{THz}$. Individual simulation results (gray traces) and the calculated mean from the ensemble (black line) are shown. The associated calculated coherence as defined in [79] is plotted on the right axis. (a2) Zoomed portion of the central part of the comb-like SC spectrum is displayed. Details of the numerical simulations are provided in ref [72]. (b) Experimental set-up for the seeded generation of a continuum at telecommunication wavelengths. (c) Resulting experimental continuum recorded after $90 \mathrm{~m}$ of highly nonlinear fiber. Details of the experimental parameters can be found in [73].

\section{Observation of the Peregrine soliton in optical fiber}

The control of MI dynamics shows that a judicious choice of excitation parameters and fibers can significantly improve the overall performance level of a broadband source by limiting the emergence of random rogue solitons. Significantly, the same kind of control can be used to generate the ultimate prototype of nonlinear rogue wave, the Peregrine soliton. This nonlinear structure has been first theoretically predicted in 1983 in hydrodynamics [11] but, up to 2010 and 
experiments in fiber optics, has never been demonstrated experimentally in any continuous physical media. The Peregrine soliton represents a limiting case of the Akhmediev breather for which the temporal localization is maximum [80]. Consequently, this pulse over a continuous background has the particularity to appear from nowhere and to disappear without leaving a trace, thus constituting an excellent prototype of oceanic rogue waves. From an experimental point of view we have shown that a sinusoidal beating created by the temporal overlap of two continuous wave that are frequency shifted [81] or the direct intensity modulation of a continuous wave [82] can reshape into a Peregrine soliton [83]. The obtained Peregrine soliton was accurately characterized using a frequency resolved optical gating device [81] or an optical sampling oscilloscope [82]. Experimental results are well reproduced both numerically and analytically as can be seen in Fig. 9. The longitudinal study performed by a cut-back technique has confirmed the strong spatial localization (Fig. 9(b)), though some replicas are manifested for large propagation distances. Those replicas can be theoretically interpreted in terms of higherorder breather solutions through the Darboux transformations and linked to the process of higher order modulation instabilities [84]. Note that the Peregrine is only the first order solution of a wider class of rational solitons that have been also numerically found in turbulent optical environments $[85 ; 86]$. 

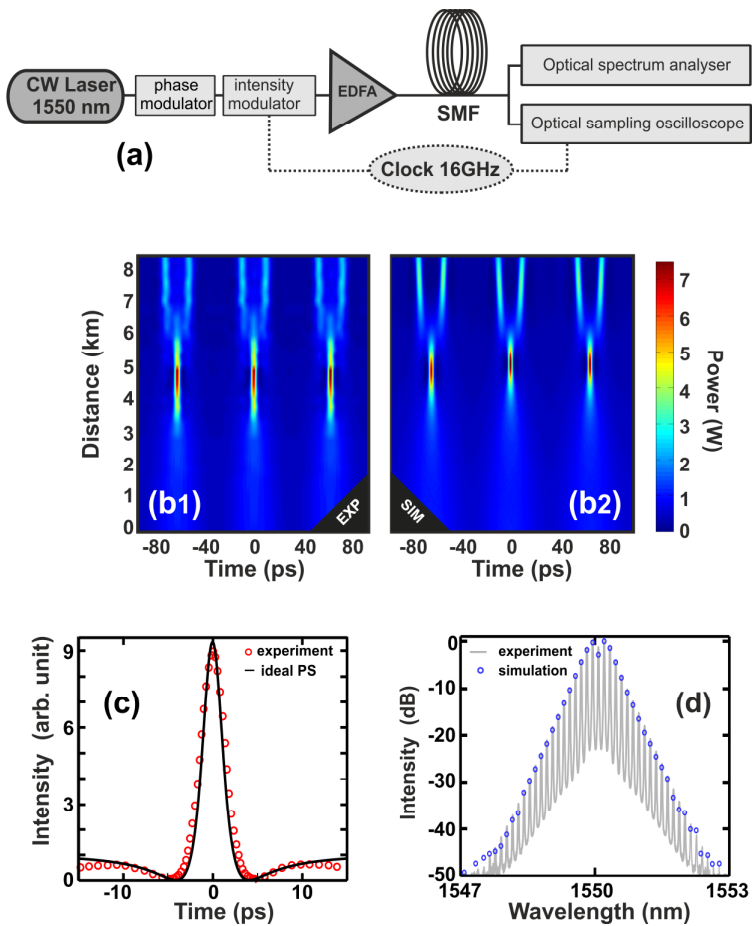

Figure 9: (a) Experimental set-up. CW : continuous wave, EDFA : erbium doped fiber amplifier, SMF : standard Single Mode Fiber ITU G652. Details of the experimental parameters are provided in [82]. (b) Longitudinal evolution of the temporal intensity profile as measured on the ultrafast sampling oscilloscope. Experiments (b1) are compared with numerical simulation of the NLSE (b2). (c) Temporal intensity profile at the point of maximum compression. The experimental results (red circles) are compared with the analytical form of the Peregrine soliton (solid black line). (d) Spectral intensity profile at the point of maximum compression. The experimental results (solid line) are compared to the results of numerical simulations (blue circles).

\section{Conclusions}

We have provided an overview of several recent results on the stability of supercontinua and associated dynamics in normally and anomalously dispersive optical fibers. Normally dispersive fibers are very attractive for the generation of stable continua and different choices of input pulse characteristics enable a rich variety of reshaping processes within the fiber. In fibers with anomalous dispersion or fibers where spontaneous modulation instability processes are significant, the stability is much more difficult to control and the resulting SC is typically 
affected by large shot-to-shot fluctuations that favor the emergence of intense solitons on the red side of the spectrum and nonlinear pulse collisions. A fine analysis of the onset of the continuum generation has demonstrated that the Akhmediev breather formalism can provide new insight to understand and predict the early stages of propagation. Moreover, seeding the SC generation process with a suitably chosen continuous signal significantly improves the stability. The same concept can be applied to stimulate the generation of the Peregrine soliton, a limiting case of the Akhmediev breather, whose temporal and spatial localizations make it an excellent prototype of rogue waves that appear from nowhere and disappear without leaving a trace. More generally, the results presented in this paper are a further illustration of how optical fiber systems can provide useful experimental testbeds for the study of complex statistical hydrodynamics problems.

\section{Acknowledgements}

We would like to thank Antonio Picozzi, Miro Erkintalo, Frederic Dias, Nail N. Akhmediev for fruitful collaboration on rogue waves, Brandon G. Bale and Sergei Turitsyn for contributing to some aspects of pulse shaping in normally dispersive fibers, Francesca Parmigiani, Lionel Provost, Periklis Petroupoulos and Dave J. Richardson for their contribution to pulse shaping in normally dispersive fibers. This research was supported by the Agence Nationale de la Recherche (ANR MANUREVA, IMFINI, SUPERCODE, SOFICARS projects), by the Conseil Régional de Bourgogne (PARI Photcom and FABER of B. Kibler), by the FEDER, by the Ministère de l'Enseignement Supérieur et de la Recherche and by the Ministère des affaires étrangères (Alliance Scheme). The experimental work carried out at telecommunication wavelengths has benefited from the PICASSO Platform equipment. 


\section{References}

[1] T. Morioka, K. Mori, S. Kawanishi, and M. Saruwatari, Pulse-width tunable, self-frequency conversion of short optical pulses over $200 \mathrm{~nm}$ based on supercontinuum generation. Electron. Lett. 30 (1994) 1960-1961.

[2] J.K. Ranka, R.S. windeler, and A.J. Stentz, Visible continuum generation in air-silica microstructure optical fibers with anomalous dispersion at $800 \mathrm{~cm}$. Opt. Lett. 25 (2000) 25-27.

[3] I. Hartl, X.D. Li, C. Chudoba, R.K. Ghanta, T.H. Ko, J.G. Fujimoto, J.K. Ranka, and R.S. Windeler, Ultrahigh-resolution optical coherence tomography using continuum generation in an air-silica microstructure optical fiber. Opt. Lett. 26 (2001) 608-610.

[4] R. Holzwarth, T. Udem, T.W. Hänsch, J.C. Knight, W.J. Wadsworth, and P.S.J. Russell, Optical Frequency Synthesizer for Precision Spectroscopy. Phys. Rev. Lett. 85 (2000) 2264-2267.

[5] M. Nakazawa, K. Tamura, H. Kubota, and E. Yoshida, Coherence degradation in the process of supercontinuum generation in an optical fiber. Opt. Fiber. Technol. 4 (1998) 215-223.

[6] D.R. Solli, C. Ropers, P. Koonath, and B. Jalali, Optical rogue waves. Nature 450 (2007) 1054.

[7] A. Hasegawa, and F. Tappert, Transmission of stationary nonlinear optical pulses in dispersive dielectric fibers. I. Anomalous dispersion. Appl. Phys. Lett. 23 (1973) 142144.

[8] D.V. Skryabin, and A.V. Gorbach, Looking at a soliton through the prism of optical supercontinuum. Rev. Modern Physics 82 (2010) 1287-1299.

[9] N.N. Akhmediev, and V.I. Korneev, Modulation instability and periodic-solutions of the nonlinear Schrödinger equation. Theor. Math. Phys. 69 (1986) 1089-1093.

[10] J.M. Dudley, G. Genty, F. Dias, B. Kibler, and N. Akhmediev, Modulation instability, Akhmediev Breathers and continuous wave supercontinuum generation. Opt. Express 17 (2009) 21497-21508. 
[11] D.H. Peregrine, Water waves, nonlinear Schrödinger equations and their solutions. J. Austral. Math. Soc. Ser. B 25 (1983) 16-43.

[12] J.M. Dudley, G. Genty, and S. Coen, Supercontinuum generation in photonic crystal fiber. Rev. Modern Physics 78 (2006) 1135-1184.

[13] J.M. Dudley, and J.R. Taylor, Supercontinuum generation in optical fibers, Cambridge University Press, 2010.

[14] G.P. Agrawal, Nonlinear Fiber Optics, Fourth Edition, Academic Press, San Francisco, CA, 2006.

[15] S.V. Smirnov, J.-D. Ania Castanon, T.J. Ellingham, S.M. Kobtsev, S. Kukarin, and S.K. Turitsyn, Optical spectral broadening and supercontinuum generation in telecom applications. Opt. Fiber. Technol. 12 (2006) 122-147.

[16] L. Boivin, and B.C. Collings, Spectrum slicing of coherent sources in optical communications. Opt. Fiber. Technol. 7 (2001) 1-20.

[17] S. Boscolo, and C. Finot, Nonlinear pulse shaping in fibres for pulse generation and optical processing. International Journal of Optics (2012) 159057.

[18] R.H. Stolen, and Q. Lin, Self-phase modulation in silica optical fibers. Phys. Rev. A 17 (1978) 1448-1453.

[19] S. Taccheo, and L. Boivin, Investigation and design rules of supercontinuum sources for WDM applications, Optical Fiber Communication, 2000, pp. ThA1.

[20] C. Finot, B. Kibler, L. Provost, and S. Wabnitz, Beneficial impact of wave-breaking on coherent continuum formation in normally dispersive nonlinear fibers. J. Opt. Soc. Am. B 25 (2008) 1938-1948.

[21] D. Anderson, M. Desaix, M. Lisak, and M.L. Quiroga-Teixeiro, Wave-breaking in nonlinear optical fibers. J. Opt. Soc. Am. B 9 (1992) 1358-1361.

[22] W.J. Tomlinson, R.H. Stolen, and A.M. Johnson, Optical wave-breaking of pulses in nonlinear optical fibers. Opt. Lett. 10 (1985) 457-459.

[23] F. Parmigiani, C. Finot, K. Mukasa, M. Ibsen, M.A.F. Roelens, P. Petropoulos, and D.J. Richardson, Ultra-flat SPM-broadened spectra in a highly nonlinear fiber using parabolic pulses formed in a fiber Bragg grating. Opt. Express 14 (2006) 7617-7622. 
[24] A.M. Clarke, D.G. Williams, M.A.F. Roelens, and B.J. Eggleton, Reconfigurable Optical Pulse Generator Employing a Fourier-Domain Programmable Optical Processor. J. Lightw. Technol. 28 (2010) 97-103.

[25] N. Verscheure, and C. Finot, Pulse doubling and wavelength conversion through triangular nonlinear pulse reshaping. Electron. Lett. 47 (2011) 1194-1196.

[26] F. Parmigiani, M. Ibsen, T.T. Ng, L. Provost, P. Petropoulos, and D.J. Richardson, An efficient wavelength converter exploiting a grating based saw-tooth pulse shaper. IEEE Photon. Technol. Lett. 20 (2008) 1461-1463.

[27] A. Latkin, S. Boscolo, R.S. Bhamber, and S.K. Turitsyn, Doubling of optical signals using triangular pulses. J. Opt. Soc. Am. B 26 (2009) 1492-1496.

[28] S. Boscolo, A.I. Latkin, and S.K. Turitsyn, Passive nonlinear pulse shaping in normally dispersive fiber systems. IEEE J. Quantum Electron. 44 (2008) 1196-1203.

[29] S.A. Planas, N.L. Pires Mansur, C.H. Brito Cruz, and H.L. Fragnito, Spectral narrowing in the propagation of chirped pulses in single-mode fibers. Opt. Lett. 18 (1993) 699-701.

[30] E.R. Andresen, J.M. Dudley, C. Finot, D. Oron, and H. Rigneault, Transform-limited spectral compression by self-phase modulation of amplitude shaped pulses with negative chirp. Opt. Lett. 36 (2011) 707-709.

[31] N.L. Markaryan, L.K. Muradyan, and T.A. Papazyan, Spectral compression of ultrashort laser pulses. Kvantovaya Elektronika 18 (1991) 865-867.

[32] J. Fatome, B. Kibler, E.R. Andresen, H. Rigneault, and C. Finot, All-fiber spectral compression of picosecond pulses at telecommunication wavelength enhanced by amplitude shaping. submitted to Applied Optics (2012).

[33] Z. Yousoff, P. Petropoulos, F. Furusawa, T.M. Monro, and D.J. Richardson, A 36-channel x $10-\mathrm{GHz}$ spectrally sliced pulse source based on supercontinuum generation in normally dispersive highly nonlinear holey fiber. IEEE Photon. Technol. Lett. 15 (2003) 16891691.

[34] S. Pitois, C. Finot, J. Fatome, and G. Millot, Generation of 20-Ghz picosecond pulse trains in the normal and anomalous dispersion regimes of optical fibers. Opt. Commun. 260 (2006) 301-306.

[35] K.J. Blow, N.J. Doran, and B.P. Nelson, All-fiber pulse compression at 1,32 um. Opt. Lett. 10 (1985) 393-395. 
[36] C. Billet, J.M. Dudley, N. Joly, and J.C. Knight, Intermediate asymptotic evolution and photonic bandgap fiber compression of optical similaritons around $1550 \mathrm{~nm}$. Opt. Express 13 (2005) 3236-3241.

[37] W.J. Tomlinson, R.H. Stolen, and C.V. Shank, Compression of optical pulses chirped by self-phase modulation in fibers. J. Opt. Soc. Am. B 1 (1984) 139-149.

[38] C.V. Shank, R.L. Fork, R. Yen, R.H. Stolen, and W.J. Tomlinson, Compression of femtosecond optical pulses. Appl. Phys. Lett. 40 (1982) 761-763.

[39] B.G. Bale, S. Boscolo, K. Hammani, and C. Finot, Effects of fourth-order fiber dispersion on ultrashort parabolic optical pulses in the normal dispersion regime. J. Opt. Soc. Am. B 28 (2011) 2059-2065.

[40] S. Demmler, J. Rothhardt, A.M. Heidt, A. Hartung, E.G. Rohwer, H. Bartelt, J. Limpert, and A. Tünnermann, Generation of high quality, 1.3 cycle pulses by active phase control of an octave spanning supercontinuum. Opt. Express 19 (2011) 20151-20158.

[41] L.E. Hooper, P.J. Mosley, A.C. Muir, W.J. Wadsworth, and J.C. Knight, Coherent supercontinuum generation in photonic crystal fiber with all-normal group velocity dispersion. Opt. Express 19 (2011) 4902-4907.

[42] M.E. Fermann, V.I. Kruglov, B.C. Thomsen, J.M. Dudley, and J.D. Harvey, Self-similar propagation and amplification of parabolic pulses in optical fibers. Phys. Rev. Lett. 84 (2000) 6010-6013.

[43] Y. Ozeki, Y. Takushima, K. Aiso, and K. Kikuchi, High repetition-rate similariton generation in normal dispersion erbium-doped fiber amplifiers and its application to multi-wavelength light sources. IEICE Trans. Electron. 88 (2005) 904-911.

[44] C. Finot, J.M. Dudley, B. Kibler, D.J. Richardson, and G. Millot, Optical parabolic pulse generation and applications. IEEE J. Quantum Electron. 45 (2009) 1482-1489.

[45] K.L. Corwin, N.R. Newbury, J.M. Dudley, S. Coen, D. S.A., K. Weber, and R.S. Windeler, Fundamental noise limitations to supercontinuum generation in microstructure fibers. Phys. Rev. Lett. 90 (2003) 113904.

[46] J. Kasparian, P. Bejot, J.P. Wolf, and J.M. Dudley, Optical rogue wave statistics in laser filamentation. Opt. Express 17 (2009) 12070-12075.

[47] K. Hammani, C. Finot, and A. Picozzi, Extreme statistics in Raman fiber amplifier : from analytical description to experiments. Opt. Commun. 284 (2010) 2594-2603. 
[48] K. Hammani, C. Finot, and G. Millot, Emergence of extreme events in fiber-based parametric processes driven by a partially incoherent wave. Opt. Lett. 34 (2009) 11381140.

[49] D. Majus, V. Jukna, E. Pileckis, G. Valiulis, and A. Dubietis, Rogue-wave-like statistics in ultrafast white-light continuum generation in sapphire. Opt. Express 19 (2011) 1631716323.

[50] S. Vergeles, and S.K. Turitsyn, Optical rogue waves in telecommunication data streams. Phys. Rev. A 83 (2011) 061801.

[51] D.V. Churkin, O. Gorbunov, and S.V. Smirnov, Extreme value statistics in Raman fiber lasers. Opt. Lett. in press (2011).

[52] A. Zaviyalov, O. Egorov, R. Iliew, and F. Lederer, Rogue waves in mode-locked fiber lasers. Phys. Rev. A 85 (2012).

[53] V. Odent, M. Taki, and E. Louvergneaux, Experimental spatial rogue patterns in an optical feedback system. Nat. Hazards Earth Syst. Sci. 10 (2010) 2727-2732.

[54] M.G. Kovalsky, A.A. Hnilo, and J.R. Tredicce, Extreme events in the Ti:sapphire laser. Opt. Lett. 36 (2011) 4449-4451.

[55] J.D. Harvey, R. Leonhardt, S. Coen, G.K.L. Wong, J.C. Knight, W.J. Wadsworth, and J.S. Russell, Scalar modulation instability in the normal dispersion regime by use of a photonic crystal fiber. Opt. Lett. 28 (2003) 2225-2227.

[56] S. Pitois, and G. Millot, Experimental observation of a new modulational instability spectral window induced by fourth-order dispersion in a normally dispersive single-mode optical fiber. Opt. Commun. 226 (2003) 415-422.

[57] B. Kibler, C. Finot, and J.M. Dudley, Soliton and rogue wave statistics in supercontinuum generation in photonic crystal fibre with two zero dispersion wavelengths. Eur. Phys. J. Special Topics 173 (2009) 289-295.

[58] K. Hammani, C. Finot, B. Kibler, and G. Millot, Soliton generation in a microstructured fiber by fourth order scalar modulation instability. IEEE Photon. J. 1 (2009) 205-212.

[59] F.M. Mitschke, and L.F. Mollenauer, Discovery of the soliton self-frequency shift. Opt. Lett. 11 (1986) 659-661. 
[60] J.M. Dudley, C. Finot, G. Millot, J. Garnier, G. Genty, D. Agafontsev, and F. Dias, Extreme events in optics: Challenges of the MANUREVA project. Eur. Phys. J. Special Topics 185 (2010) 125-133.

[61] C. Kharif, E. Pelinovsky, and A. Slunyaev, Rogue waves in the ocean, Springer, 2009.

[62] M. Taki, A. Mussot, A. Kudlinski, E. Louvergneaux, M.I. Kolobov, and M. Douay, Thirdorder dispersion for generating optical rogue solitons. Phys. Lett. A 374 (2010) 691-695.

[63] C.H. Hage, B. Kibler, and C. Finot, Fiber-based device for the detection of low intensity fluctuations of ultrashort pulses. Appl. Opt. 51 (2012).

[64] M. Erkintalo, G. Genty, and J.M. Dudley, On the statistical interpretation of optical rogue waves. Eur. Phys. J. Special Topics 185 (2010) 135-144.

[65] C. Liu, E.J. Rees, T. Laurila, S. Jian, and C.F. Kaminski, An adaptive filter for studying the life cycle of optical rogue waves. Opt. Express 18 (2010) 26113-26122.

[66] G. Genty, C.M. De Sterke, O. Bang, F. Dias, N. Akhmediev, and J.M. Dudley, Collisions and turbulence in optical rogue wave formation. Phys. Lett. A 374 (2010) 989-996.

[67] K. Hammani, B. Kibler, C. Finot, and A. Picozzi, Emergence of rogue waves from optical turbulence. Phys. Lett. A 374 (2010) 3585-3589.

[68] M. Erkintalo, G. Genty, and J.M. Dudley, Experimental signatures of dispersive waves emitted during soliton collisions. Opt. Express 18 (2010) 13379.

[69] N. Nishizawa, and T. Goto, Pulse trapping by ultrashort soliton pulses in optical fibers across zero-dispersion wavelength. Opt. Lett. 27 (2002) 152-154.

[70] A. Kudlinski, B. Barviau, A. Leray, C. Spriet, L. Héliot, and A. Mussot, Control of pulse-topulse fluctuations in visible supercontinuum. Opt. Express 18 (2010) 27445-27454.

[71] J.M. Dudley, G. Genty, and B.J. Eggleton, Harnessing and control of optical rogue waves in supercontinuum generation Opt. Express 16 (2008) 3644-3651.

[72] G. Genty, and J.M. Dudley, Route to coherent supercontinuum generation in the long pulse regime. IEEE J. Quantum Electron. 45 (2009) 1331-1335.

[73] B. Barviau, C. Finot, J. Fatome, and G. Millot, Generation from continuous waves of frequency combs with large overall bandwidth and tunable central wavelength. Electronics Letters 43 (2007) 886-887.

[74] D.R. Solli, C. Ropers, and B. Jalali, Active control of Rogue Waves for stimulated supercontinuum generation. Phys. Rev. Lett. 101 (2008) 233902. 
[75] K.Y. Cheung, C. Zhang, Y. Zhou, K.Y. Wong, and K.K. Tsia, Manipulating supercontinuum generation by minute continuous wave. Opt. Lett. 36 (2011) 160-162.

[76] D.R. Solli, B. Jalali, and C. Ropers, Seeded Supercontinuum Generation with Optical Parametric Down-Conversion. Phys. Rev. Lett. 105 (2010) 233902.

[77] J. Fatome, S. Pitois, C. Fortier, B. Kibler, C. Finot, G. Millot, C. Courde, M. Lintz, and E. Samain, Multiple four-wave mixing in optical fibers : 1.5-3.4-THz femtosecond pulse sources and real-time monitoring of a 20-GHz picosecond source. Opt. Commun. 283 (2010) 2425-2429.

[78] K. Hammani, B. Wetzel, B. Kibler, J. Fatome, C. Finot, G. Millot, N. Akhmediev, and J.M. Dudley, Spectral dynamics of modulation instability described using Akhmediev breather theory. Opt. Lett. 36 (2011) 2140-2142.

[79] J.M. Dudley, and S. Coen, Numerical simulations and coherence properties of supercontinuum generation in photonic crystal and tapered optical fibers. IEEE J. Sel. Top. Quantum Electron. 8 (2002) 651-659.

[80] N. Akhmediev, A. Ankiewicz, and M. Taki, Waves that appear from nowhere and disappear without a trace. Phys. Lett. A 373 (2009) 675-678.

[81] B. Kibler, J. Fatome, C. Finot, G. Millot, F. Dias, G. Genty, N. Akhmediev, and J.M. Dudley, The Peregrine soliton in nonlinear fibre optics. Nature Physics 6 (2010) 790-795.

[82] K. Hammani, B. Kibler, C. Finot, P. Morin, J. Fatome, J.M. Dudley, and G. Millot, Peregrine soliton generation and breakup in standard telecommunications fiber. Opt. Lett. 36 (2011) 112-114.

[83] M. Erkintalo, G. Genty, B. Wetzel, and J.M. Dudley, Akhmediev breather evolution in optical fiber for realistic initial conditions. Phys. Lett. A 375 (2011) 2029-2034.

[84] M. Erkintalo, K. Hammani, B. Kibler, C. Finot, N. Akhmediev, J.M. Dudley, and G. Genty, Higher-Order Modulation Instability in Nonlinear Fiber Optics. Phys. Rev. Lett. 107 (2011) 253901.

[85] N. Akhmediev, J.M. Soto-Crespo, and A. Ankiewicz, Extreme waves that appear from nowhere : on the nature of rogue waves. Phys. Lett. A 373 (2009) 2137-2145.

[86] B. Kibler, K. Hammani, C. Michel, C. Finot, and A. Picozzi, Rogue waves, rational solitons and wave turbulence theory. Phys. Lett. A 375 (2011) 3149-3155. 
